\title{
Phonon scattering and exciton localization: molding exciton flux in two dimensional disorder energy landscape
}

\author{
Pengfei Qi ${ }^{1}{ }^{2 \dagger}$, Yang Luo ${ }^{1 \dagger}$, Beibei Shi ${ }^{1}$, Wei Li ${ }^{3}$, Donglin Liu' ${ }^{1}$ Liheng Zheng ${ }^{1}$, Zhixin Liu' ${ }^{1}$, Yanglong Hou ${ }^{3}$ and \\ Zheyu Fang ${ }^{1 *}$
}

\begin{abstract}
Two dimensional excitonic devices are of great potential to overcome the dilemma of response time and integration in current generation of electron or/and photon based systems. The ultrashort diffusion length of exciton arising from ultrafast relaxation and low carrier mobility greatly discounts the performance of excitonic devices. Phonon scattering and exciton localization are crucial to understand the modulation of exciton flux in two dimensional disorder energy landscape, which still remain elusive. Here, we report an optimized scheme for exciton diffusion and relaxation dominated by phonon scattering and disorder potentials in $\mathrm{WS}_{2}$ monolayers. The effective diffusion coefficient is enhanced by $>200 \%$ at $280 \mathrm{~K}$. The excitons tend to be localized by disorder potentials accompanied by the steadily weakening of phonon scattering when temperature drops to $260 \mathrm{~K}$, and the onset of exciton localization brings forward as decreasing temperature. These findings identify that phonon scattering and disorder potentials are of great importance for long-range exciton diffusion and thermal management in exciton based systems, and lay a firm foundation for the development of functional excitonic devices.
\end{abstract}

Keywords: Exciton diffusion, Exciton relaxation, 2D semiconductors, Phonon scattering, Disorder potentials

\section{Introduction}

Excitons, or bound electron-hole pairs, can be deeply compatible with current generation of optoelectronic system [1]. As hydrogen-like bosonic quasiparticles with Bohr radius of nanometer dimension, excitons combine the advantages of electrons and photons. Therefore, elements and devices operating with excitons have promising prospects for overcoming the dilemma of response time and integration in electron or/and photon based system [1-3]. Meanwhile, owing to reduced dielectric screening, enhanced Coulomb interactions and relatively

\footnotetext{
*Correspondence: zhyfang@pku.edu.cn

†Pengfei Qi and Yang Luo contributed equally to this work

${ }^{1}$ School of Physics, State Key Laboratory for Mesoscopic Physics,

Academy for Advanced Interdisciplinary Studies, Collaborative Innovation Center of Quantum Matter, Nano-Optoelectronics Frontier Center of Ministry of Education, Peking University, Beijing 100871, China Full list of author information is available at the end of the article
}

large effective masses of charge carriers, transition metal dichalcogenide (TMDC) monolayers can provide a great platform for manipulating excitons at room temperature, where the excitons have $\sim 1 \mathrm{~nm}$ Bohr radius and $\sim 500 \mathrm{meV}$ binding energy - over an order of magnitude larger than conventional semiconductors [4-7]. Therefore, excitonic functional devices based on TMDC monolayers at room temperature have attracted intense interests in past decades, owing to the advantages of high operation and interconnection speed, small dimensions and the opportunity to highly integrate [8-13].

The imperative step towards excitonic devices is the comprehension and manipulation of exciton transport dynamics [14-16]. As is known, exciton flux can be effectively modulated by strain, electric field, electrondoping, local dielectric environment, grain boundaries, moiré potentials, etc. [17-25]. However, the ultra-short lifetimes and low carrier mobility of excitons in TMDC 
monolayers give rise to extremely low exciton diffusion coefficient, which critically limits the development of excitonic devices. Currently, the exciton diffusion length of $2 \mu \mathrm{m}$ was obtained by utilizing ultrahigh excitation power to improve the effective diffusion coefficient [26]. On the other hand, exciton diffusion length could be improved to $5 \mu \mathrm{m}$ for the interlayer excitons with longer lifetime [19]. Although we demonstrated the remote lightening of $14.6 \mu \mathrm{m}$ via exciton flux by femtosecond excitation source and bright emitter [23], the estimated effective diffusion coefficient is still on the scale of 10 $\mathrm{cm}^{2} / \mathrm{s}$ [26]. Therefore, the long-range exciton transport still remains challenging on the road to excitonic devices.

Phonon scattering is crucial to understand the carrier transport phenomena in quantum confined systems [27-31]. It is conceivable that the mobility of carriers including electron, hole and exciton can be improved by refrigerating to suppress phonon scattering $[29,31]$. However, disorder potentials induced by base and sample surface imperfection will lead to carrier localization, providing thermal fluctuations can't support carrier escape. Even to the high-quality monolayer flakes exfoliated from layered bulk crystals [32-34], the imperceptible fluctuations generating from exfoliation and transfer still can impose disordered potentials on monolayer flakes at cryogenic temperature [35-39]. Consequently, exploring the nonequilibrium dynamics of two dimensional (2D) excitons modulated by phonon scattering and disorder potentials is of great significance for long-range exciton diffusion and thermal management in exciton based systems.

Here, nonequilibrium dynamics of $2 \mathrm{D}$ exciton modulated by phonon scattering and disorder potentials are clarified. Most intriguingly, exciton diffusion coefficient is non-monotonous with temperature variation, and exciton ultrafast relaxation can be effectively modulated. The underlying mechanism is the competition of phonon scattering and disorder potentials, which is further confirmed by photoluminescence (PL) spectra. It indicates that disorder potentials are still crucial to understand exciton dynamics of high-quality monolayer flakes at cryogenic temperature. Our results present evidences that phonon scattering and disorder potentials should be optimized for long-range exciton diffusion and thermal management in exciton based system.

\section{Results and discussion}

\subsection{Characterization of mechanically exfoliated $\mathrm{WSe}_{2}$ monolayer}

In this study, $\mathrm{WSe}_{2}$ monolayers were mechanically exfoliated from layered bulk crystals by polydimethylsiloxane (PDMS) and then transferred to silicon wafers. All our measurements in this part were performed at room temperature. The $\mathrm{WSe}_{2}$ monolayers were confirmed by microscopy and PL spectroscopy. Figure 1a illustrates a typical optical microscope image of $\mathrm{WSe}_{2}$ monolayer, and the linear dimensions of areas with uniform monolayer flake are in the range from $\sim 20 \mu \mathrm{m}$ to well above $50 \mu \mathrm{m}$. Combining confocal optical microscopy with spectrograph, excitation laser (continuous wave, $2.33 \mathrm{eV}$ ) was scanned over the sample and the PL emission spectra at different sites were recorded. Figure $1 \mathrm{~b}$ shows the spatial distribution of total PL intensity, where the quantum efficiency of PL is homogeneous over the exfoliated $\mathrm{WSe}_{2}$ monolayer. The PL spectra along the white dotted line in Fig. 1b were depicted in Fig. 1c. There is no appreciable shift for exciton peak. Moreover, the average PL spectrum of $\mathrm{WSe}_{2}$ monolayer (gray line) was superimposed on Fig. 1c. The near symmetric spectral shape was well fitted by Lorentzian profile (red line) with the peak of $1.66 \mathrm{eV}$ and linewidth of $39.4 \mathrm{meV}$, which can be attributed to A exciton. It is different from chemical vapor deposition-grown TMDC monolayers, where exciton emission is often accompanied by comparable trion emission due to electrons doping from defects and grain boundaries. Therefore, defects and grain boundaries have minimal effect on our experiments.

Besides, micro-Raman spectroscopy also can serve as an effective technique to delineate the spatial distributions of defects, strain, electron-doped concentration etc. As shown in Fig. 1e, the intensities of Raman signals for exfoliated $\mathrm{WSe}_{2}$ monolayer are homogeneous, coinciding with the PL mapping in Fig. 1b. Similarly, Raman spectra along the white dotted line in Fig. 1d were depicted in Fig. 1e, superimposing with the average spectrum. The $\mathrm{WSe}_{2}$ monolayer has a typical Raman spectrum with two dominant peaks at $248.8 \mathrm{~cm}^{-1}$ and $259.8 \mathrm{~cm}^{-1}$, corresponding to atomic displacements of the in-plane $E_{2 g}^{1}$ and out-of-plane $A_{1 g}$ modes, respectively. The shift magnitudes of in-plane mode in different regions are often utilized to determine defects and strain distributions [40, 41]. The $E_{2 g}^{1}$ peak frequencies of Fig. 1d were extracted and depicted in Figs. 1f, where there is no discernible shift for $E_{2 g}^{1}$ mode, corroborating the uniformity of $\mathrm{WSe}_{2}$ monolayer. These findings suggest that mechanically exfoliated $\mathrm{WSe}_{2}$ monolayers can provide an ideal platform to explore the diffusion dynamics of $2 \mathrm{D}$ exciton.

\subsection{Exciton diffusion dynamics modulated by phonon scattering and disorder potentials}

The direct PL imaging technique was adopted to explore exciton diffusion dynamics modulated by phonon scattering and disorder potentials in the exfoliated $\mathrm{WSe}_{2}$ monolayer. Therefore, only the diffusion of bright exciton within the radiative cone can be recorded and discussed in this study, though the population of dark excitons 


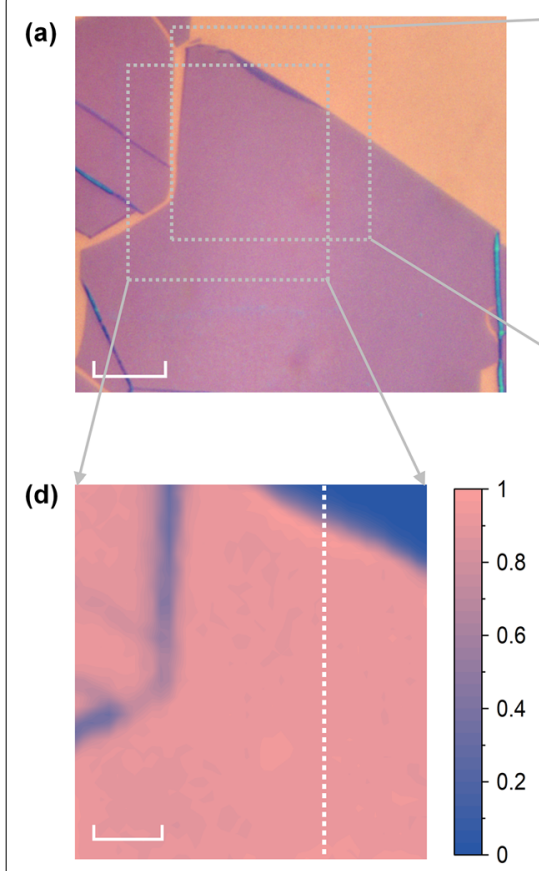

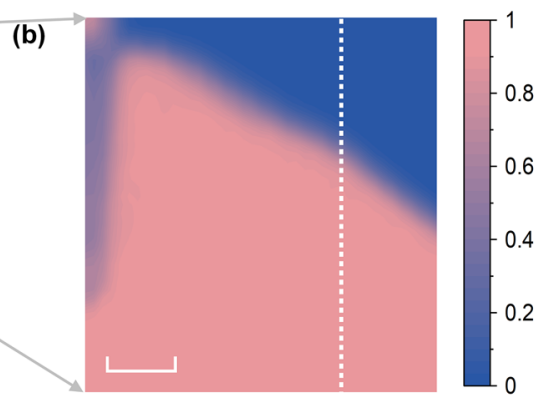

(c)

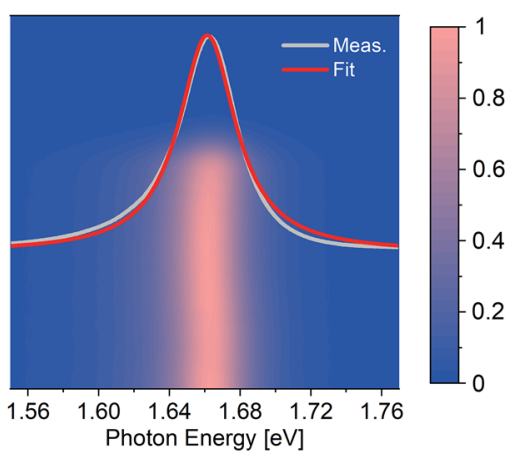

(e)
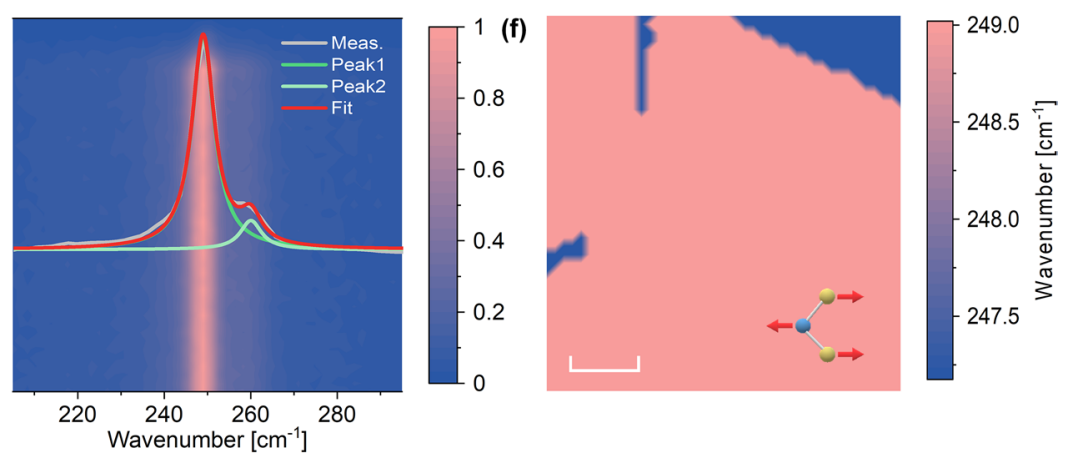

Fig. 1 Characterization of mechanically exfoliated WSe $e_{2}$ monolayer. a Optical microscope image and $\mathbf{b}$ PL mappings of WSe ${ }_{2}$ monolayer. c PL spectra along the white dotted line in $\mathbf{b}$, inset: average photoluminescence spectrum and fitted curve. $\mathbf{d}$ Raman mappings of WS $\mathrm{e}_{2}$ monolayer. $\mathbf{e}$ Raman spectra along the white dotted line in $\mathbf{d}$. Inset: average Raman spectrum and fitted curves. $\mathbf{f}$ Peak frequency shift of the in-plane $E_{2 g}^{1}$ mode. Inset: Schematic atomic displacement of in-plane mode. Scale bar in a is $10 \mu \mathrm{m}$, others are $5 \mu \mathrm{m}$

plays an important role in the physical properties at low temperatures [42-44]. In our experiments, the flake was excited by the focused femtosecond laser with the photon energy of $2.06 \mathrm{eV}$ and energy density of $2 \mu \mathrm{J} / \mathrm{cm}^{2}$. The focused femtosecond laser spot was normalized and depicted in Fig. 2a. The pumped excitons undergo ultrafast Auger recombination and then diffuse and recombine with bright radiation around the uniformly high-quality flake [45-47], which were recorded simultaneously by an electron-multiplying charge-coupled device (EMCCD) camera. In Fig. 2a, the recorded PL images at different temperatures were normalized and depicted in both ordinary and semi-logarithmic coordinate systems, respectively (see Additional file 1: S1 for more temperatures). It can be observed that excitons spread much further than laser spot due to diffusion.

The theoretical exciton emission profiles (Additional file 1: Fig. S2a) for different effective diffusion coefficients $D_{\text {eff }}$ can be obtained based on the continuity equation of exciton diffusion dynamics. It is noted that the excitation location was fixed in the middle of the $\mathrm{WSe}_{2}$ sample to avoid scattering and other effects induced by boundary region. To extract exciton diffusion coefficient sensitively, the co-axial ring of PL images (inset of Fig. 2b) was adopted, by imitating the commonly used eclipsing
Z-scan method in nonlinear optics [48, 49]. After determining the co-axial ring, the $D_{\text {eff }}$ can be obtained from Fig. 2a by comparing the theoretical and experimental results (see Additional file 1: S2 for more details), as shown in Fig. $2 \mathrm{~b}$ with the red dashed arrowed line as a guide to the eye. As increasing temperature from 80 to $400 \mathrm{~K}$, the effective diffusion coefficient of excitons was enhanced by $>200 \%$ at the vicinity of $280 \mathrm{~K}$ and then decrease rapidly. Additionally, the total PL intensities exhibit similar temperature dependence, as illustrated in Fig. 2c. It can be deduced that the temperature-dependent emission and diffusion of exciton originate from the same mechanism. Figure $2 \mathrm{c}$ seems in contradiction to the seminal work of Zhang et al. [50], where a clear and monotonous increase in PL intensity has been observed at enhanced temperatures due to the presence of energetically lowest dark excitons. In our work, the monotonous results were repeated for temperature lower than $280 \mathrm{~K}$. However, the PL decrease was observed for temperatures higher than $280 \mathrm{~K}$ due to the bright exciton quenched by multi-phonons processes. Noted that the main purpose of Zhang et al. was to present experimental evidence for the existence of dark exciton, thus the multi-phonons quenching processes were ignored at higher temperature, though the decrease of the populations of bright 


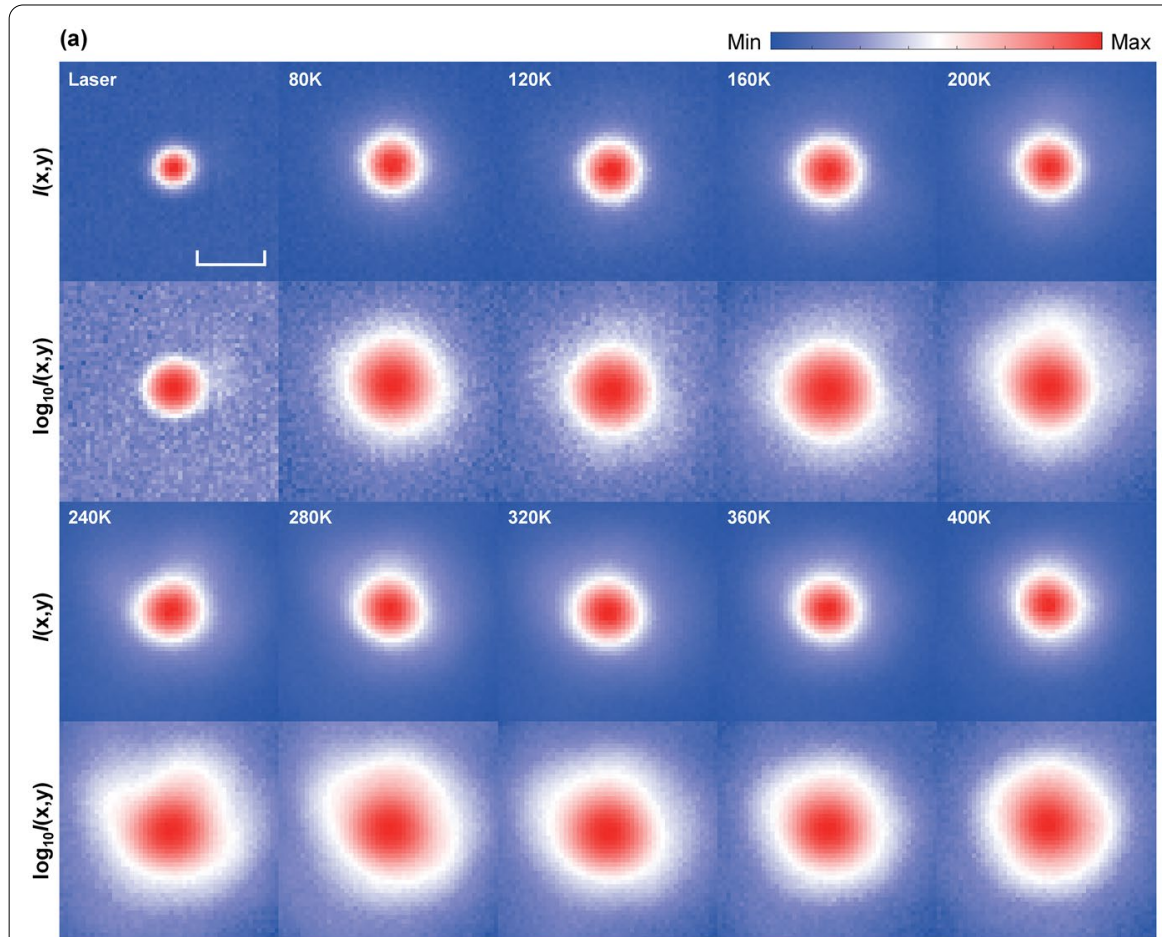

(b)

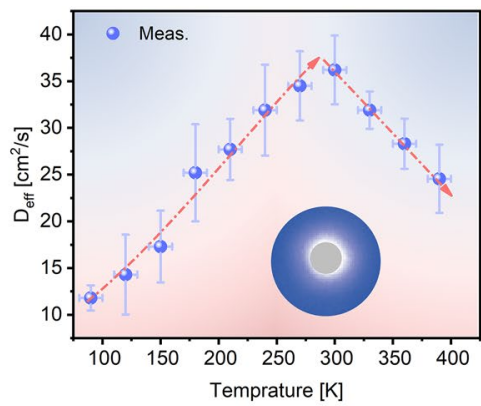

(c)

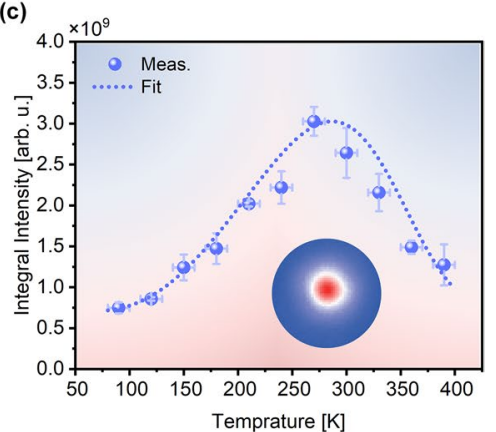

Fig. 2 Exciton diffusion dynamics modulated by phonon scattering and disorder potentials. a PL images of WSe ${ }_{2}$ monolayer for different temperatures, with the exposure time of $0.1 \mathrm{~s}$. b Temperature-dependent effective diffusion coefficient. c Temperature-dependent total PL intensity. The scale bar is $2 \mu \mathrm{m}$

state was observed for temperatures higher than $280 \mathrm{~K}$. It proves that the non-monotonous behavior of PL intensity is reliable. Besides, it should be noted that in our experiment, the energy density is in the range of halo effects, as shown in Ref. [26], but the steady state exciton distribution is recorded by EMCCD, which is the integral of time-resolved the exciton distribution. Therefore, the instantaneous halo effects weren't observed.

Taking into account the population of bright state, exciton quenching induced by multi-phonons scattering and exciton localization in disorder potentials, the non-monotonous behavior of PL intensity can be quantitatively explained. Firstly, the conduction band splitting $\Delta_{\mathrm{c}}$ results in an energy separation between bright state $\left(A_{B}\right)$ and dark state $\left(A_{D}\right)$ for $A$ exciton and the lowest energy exciton is a dark state for $\mathrm{WX}_{2}(\mathrm{X}=\mathrm{S}, \mathrm{Se}, \mathrm{Te})$. The populations of $A_{B}$ and $A_{D}$ obey Boltzmann distribution as given by $N_{A_{B}} / N_{A_{D}}=\exp \left(-\Delta_{c} / k_{B} T\right)$. Secondly, the average population of phonon gas in $\mathrm{WSe}_{2}$ monolayer is determined by temperature and can be written as $\bar{n}=1 /\left[\exp \left(\hbar \omega_{q} / k_{B} T\right)-1\right]$. Finally, the process of excitons escaped from disorder potentials is similar to electrons emission from potential wells and the probability $\eta_{e}$ is proportional to $\left(k_{B} T\right)^{1 / 2} \exp \left(-\chi / k_{B} T\right)$, where $\chi$ is the average depth of disorder potentials. Therefore, the temperature-dependent PL intensity can be written as following (see Additional file 1: S3 for more details)

$$
I_{P L}(T)=\frac{\eta_{q} \eta_{e} N_{0}}{\exp \left(\Delta_{c} / k_{B} T\right)+1} \exp \left(-\eta_{p} \bar{n}^{x} \tau\right)
$$

where $\eta_{q}$ is the quantum efficiency from excitons to photons, $N_{0}$ is the excitons population excited by photons, $\eta_{p}$ is the probability of multi-phonons collided with exciton which is proportional to $T^{x / 2}$ (here $x=4$ were adopted) and $\tau$ is exciton lifetime. As depicted by the blue dotted line in Fig. 2c, the temperature-dependent PL intensities were well fitted by Eq. (1).

Similarly, the non-monotonous temperature-dependent behavior of the exciton diffusion coefficient can be also well explained by the competition of multiphonons scattering and exciton localization in disorder potentials. According to reference [51], taking into account the interaction of exciton with longitudinal phonon mode, the quantum correction of exciton diffusion coefficient is negative and demonstrates sizeable variation with the temperature, that is, the exciton diffusion coefficient decreases with elevated temperature for the multi-phonons scattering dominated exciton diffusion. The temperature dependence of exciton 
diffusion coefficients prominently changes if the process that the excitons interacting with potential disorder is considered, and the diffusion coefficients increase with elevated temperature. Therefore, the exciton diffusion coefficients can be presented by a non-monotonic function of temperature due to the competition between multi-phonons scattering and exciton localization in disorder potentials, and the optimized temperature relates to the modulation depth of disordered potentials on the exciton energy landscape.

Note that both the measurements and calculations yield exciton diffusion coefficient in the range of $1 \sim 5$ $\mathrm{cm}^{2} / \mathrm{s}$ at room temperature under the excitation of continuous laser due to the exciton-phonon scattering in TMDC monolayers [44, 51, 52]. As demonstrated in previous studies, a significantly higher value of diffusion coefficients $D$ on the scale of $10 \mathrm{~cm}^{2} / \mathrm{s}$ can be achieved in the nonlinear regime of the exciton system, which are attributed to several possible effects including Auger-recombination, phonon drag, thermal drift (Seebeck) effects, etc. [26, 43, 53]. Specifically, the main origin of the nonlinear exciton diffusion at elevated densities, Auger recombination in the center of laser spot, leads to a flatten and additional broaden exciton distribution and thus to a giant enhancement of the extracted effective diffusion coefficient.

\subsection{Temperature-dependent photoluminescence spectra} $\mathrm{PL}$ spectra can provide the frequency domain information of exciton evolution with temperature, which is instructive to verify the physical mechanism underlying exciton diffusion. Figure 3a displays the PL spectra of different temperatures. The PL intensities were acquired by integrating spectra, as depicted in Fig. 3b. The blue dotted line is the fitted curve according to Eq. (1), which agrees well with Fig. 2c. For clarity, all the PL spectra were normalized by each maximum, as illustrated in Fig. 3c. It can be clearly observed the exciton peak broadening and red shift with rising temperature. The amplitude of exciton peak broadening and shift were extracted and plotted as the red and blue balls in Fig. 3d, respectively.

In 2D TMDCs, the variation of exciton peak can be attributed to temperature-dependent lattice dilatation and exciton-phonon interaction. As shown by the blue dotted line in Fig. 3d, the exciton peak shift was well fitted by the well-known Varshni equation, $E_{g}(T)=E_{g}(0)-\alpha T^{2} /(T+\beta)$, which describes the temperature dependence of the energy gap for various semiconductors including 2D TMDCs. [54-58]. Here, $\alpha$ is the scaling factor of the mean occupancy of an average phonon, $E_{g}(0)$ is the exciton bandgap energy at $0 \mathrm{~K}$, and $\beta$ is the average phonon temperature. The global optimal parameters of fitting curves are $E_{g}(0)=1.724 \pm 0.001 \mathrm{eV}$,

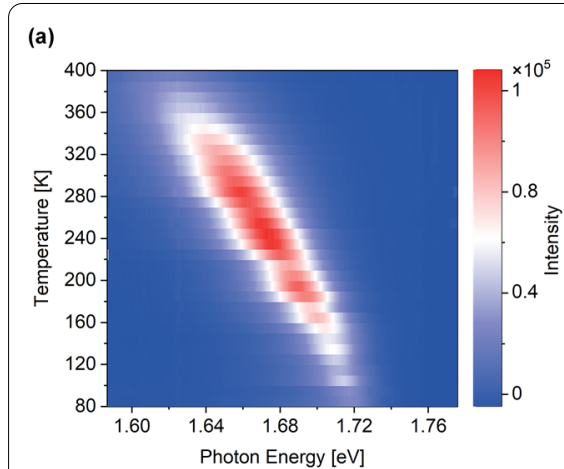

(b)

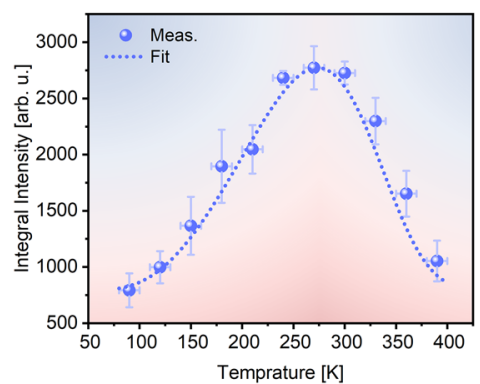

(c)

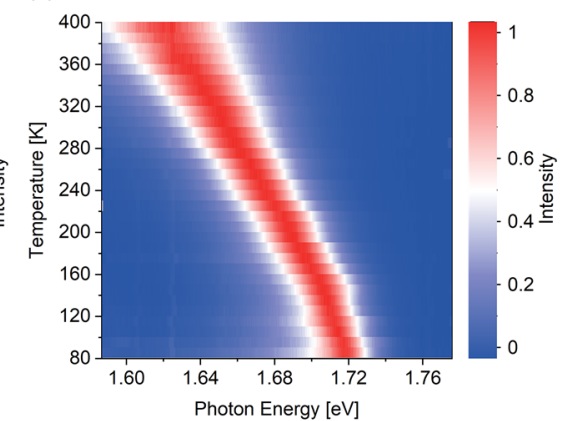

(d)

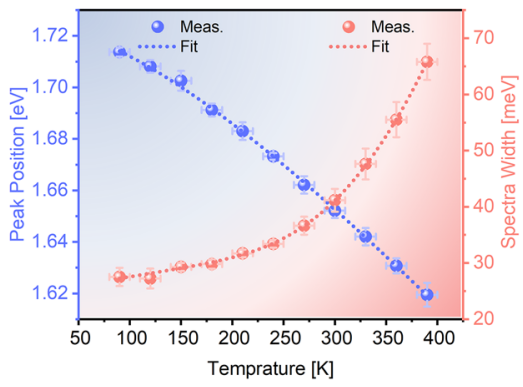

(e)

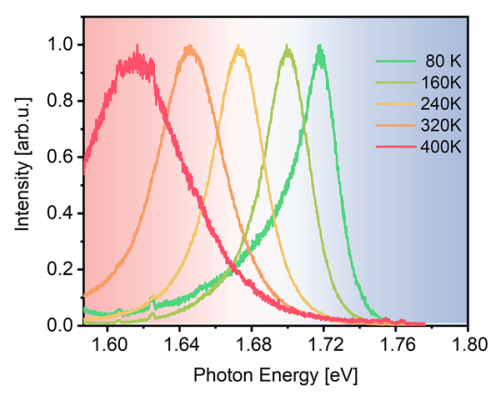

(f)

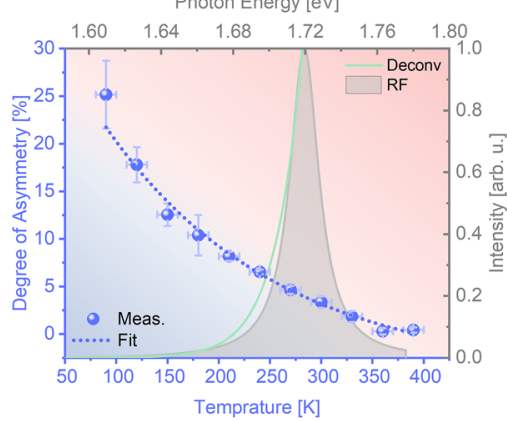

Fig. 3 Temperature-dependent PL spectra. a, b PL spectra and total intensity of exciton in WSe $\mathrm{S}_{2}$ monolayer for different temperatures. $\mathbf{c}$ Evolution of normalized spectra with temperature. $\mathbf{d}$ Evolution of peak energy and spectral width with temperature. e Normalized spectra extracted from $\mathbf{c}$. f Temperature-dependent DOA of PL spectra (blue balls) and exponential fitting (blue dotted curve), deconvolution of exponential tail for the PL spectra at $80 \mathrm{~K}$ (green line) and response function (gray curve) 
$\alpha=(4.73 \pm 0.27) \times 10^{-4} \mathrm{eV} / \mathrm{K}$ and $\beta=296 \pm 40 \mathrm{~K}$. The broadening excitons line-width results from the interaction of excitons with the longitudinal-acoustical (LA) and longitudinal-optical (LO) phonon modes of lattice for semiconductor without considerable impurity doping and defects. Accordingly, the temperature-dependent line-width of excitons can be written as $\Gamma(T)=\Gamma_{0}+\gamma_{\mathrm{LA}}$ $T+\gamma_{\mathrm{LO}} N_{\mathrm{LO}}(T)[59,60]$, where the second term describes the contribution of the interaction between excitons and LA phonons, the third term describes the interaction between excitons and LO phonons, $N_{\mathrm{LO}}(T)$ represents the LO phonons occupation with Bose-Einstein distribution, and the constant term arises from the scattering due to intrinsic imperfections. As depicted by the red dotted line in Fig. 3d, temperature-dependent line-width can be strictly described by $\Gamma(T)$, where $\Gamma_{0}=24.52 \pm 0.76 \mathrm{meV}$, $\gamma_{\mathrm{LA}}=30 \pm 4.7 \mu \mathrm{eV} / \mathrm{K}$, and $\gamma_{\mathrm{LO}}=2.72 \pm 0.92 \mathrm{eV}$. It can be concluded that the linear broaden line-width mainly stems from LA phonons, while the superlinear broaden line-width is attributed to LO phonons, and the temperature-independent offset $\Gamma_{0}$ can be ascribed to unavoidable fluctuations of samples.

In addition, the measured PL spectra gradually turn to asymmetric line shape with a sharp high-energy cutoff and an exponential low-energy tail when the temperature drops from 400 to $80 \mathrm{~K}$, as shown in Fig. 3e. To quantify the asymmetric line shape, we define degrees of asymmetry (DOA) as $\left|I_{L}-I_{H}\right| /\left|I_{L}+I_{H}\right|$, where $I_{L}$ and $I_{H}$ are the integrated intensity for the photon energy lower and higher than exciton peak. As illustrated in Fig. 3f, DOA increases rapidly from near $0 \%$ at $400 \mathrm{~K}$ to $25 \%$ at $80 \mathrm{~K}$ with an exponential trend (blue dotted line). As is well known, exciton localization and phonon sidebands are the main origins for the asymmetric line shape of PL spectra $[39,55,61]$. The temperature-dependent asymmetric line shape can help us to determine the actual origin of asymmetric line shape. For the asymmetric line shape induced by phonon sidebands, the higher the temperature, the larger is the phonon population, the more pronounced are the sidebands. Meanwhile, the asymmetric line shape presents as an exponential high-energy tail and a sharp low energy cutoff $[55,61]$. The phenomena mentioned above are remarkably reversed with our experimental results. Therefore, phonon sidebands can be reasonably excluded. Additionally, the excitation intensity dependence of the PL integrated intensity of the free exciton band and the low-energy band at $80 \mathrm{~K}$ were discussed. The contribution of the free exciton band increases gradually with the increase of excitation intensity, and the contribution of the low-energy band is contrary (see Additional file 1: S5 for more details). It can be further demonstrated that the asymmetric spectral shape mainly originates from exciton localization. Focusing on exciton localization, the exponential low-energy tail indicates the emergence of new excitonic states below existing exciton energy. The exponential low-energy tail reflects the density of new excitonic states, whereas a sharp high energy cutoff corresponds to mobility edge. Owing to the low electron-doped concentration, new excitonic states mainly stem from excitons localized by disorder potentials arising from unavoidable fluctuations of samples. It is consistent with decreased effective diffusion coefficients at cryogenic temperature. Moreover, Fig. 3e shows a PL emission with photon energy lower than that of the free exciton emission band at cryogenic temperature, which is the convolution of the intrinsic distribution of exciton energy and response function (RF, including the physical spectral broadening and instruments response) [62-64]. In order to extract the intrinsic exciton energy spectrum, deconvolution with RF (equivalent to the free exciton band) is essential, as shown in Fig. 3f. The phenomena depicted in Fig. 3 can be repeated for the excitation photon energy of $3.04 \mathrm{eV}$ (see Additional file 1: S4 for more details).

\subsection{Physical scenario of temperature-dependent exciton diffusion dynamics}

As aforementioned, the decreased diffusion coefficients and asymmetric PL spectra of excitons at cryogenic temperature were attributed to the localized excitons in disorder potentials induced by surface fluctuations. The Raman spectra of excitons have been demonstrated as an effective technique to map the subtle fluctuations of samples. The mini-strain arising from surface fluctuations has minimal effect on Raman spectra at room temperature (Fig. 1d, e). However, owing to reduced thermal fluctuations, the intensity and peak of Raman spectra at cryogenic temperature can be significantly modulated by the mini-strain distribution on the sample, as shown in Fig. $4 \mathrm{a}$ and $\mathrm{b}$. It has been proved that the in-plane mode is more sensitive to strain than the out-plane mode [18, $65,66]$. Therefore, the red-shift magnitudes of in-plane mode in different positions (Fig. 4b) enable us to estimate the mini-strain distribution of $\mathrm{WSe}_{2}$ monolayer. The formula $\varepsilon=\Delta \omega / \chi$ was generally adopted to calculate the strain strength corresponding to Raman shifts $\Delta \omega$, where $X$ is the shift rates of Raman vibrational modes [66]. The peak positions of in-plane mode induced by surface fluctuations were obtained as $249.89 \pm 0.19 \mathrm{~cm}^{-1}$ through Lorentz fitting and statistical analysis (Additional file 1: Fig. S6a), where $249.89 \mathrm{~cm}^{-1}$ and $0.19 \mathrm{~cm}^{-1}$ are mean and standard deviation, respectively. Note that the Raman spectra at room temperature and $120 \mathrm{~K}$ were performed with the same instrument and setup. For the Raman mapping at room temperature, the Raman peak frequencies of the in-plane mode are almost entirely 

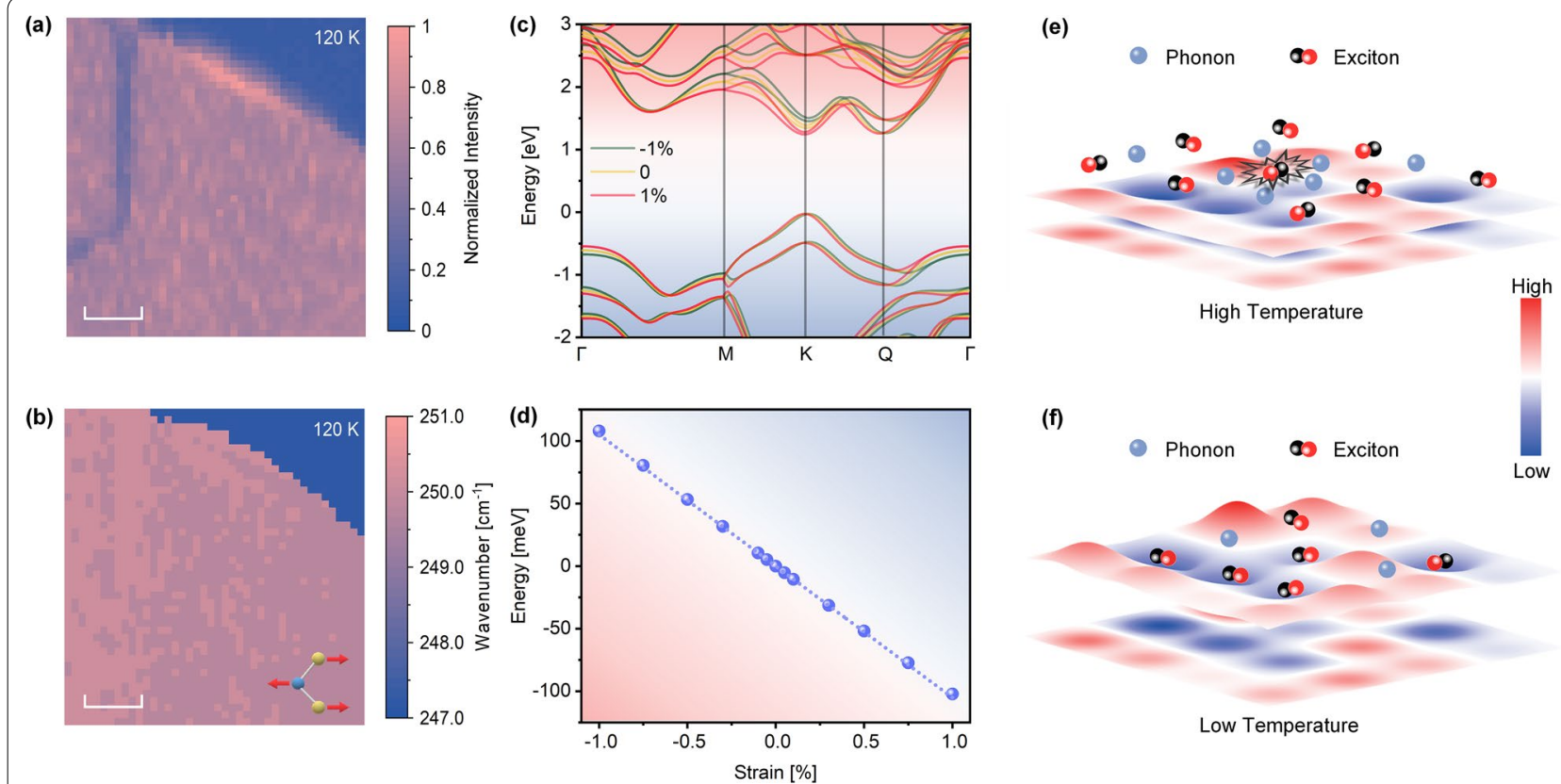

Fig. 4 Physical scenario of exciton diffusion dynamics modulated by phonon scattering and disorder potentials. a Raman mappings of WSe 2 monolayer at $120 \mathrm{~K}$. The color bar represents the normalized intensity of Raman spectra. b Peak frequency shift of in-plane Raman modes. Inset: Schematic atomic displacement of in-plane mode. c Strain-dependent electronic band structure. $\mathbf{d}$ Strain-dependent exciton energy. e, $\mathbf{f}$ Physical scenario of exciton nonequilibrium dynamics for high and low temperature, respectively. Scale bars are $5 \mu \mathrm{m}$

located at $249.02 \mathrm{~cm}^{-1}$ (Fig. 1f) for different positions of monolayer $\mathrm{WSe}_{2}$. It indicates that the standard deviation of $0.19 \mathrm{~cm}^{-1}$ (Fig. 4b) is derived from disorder potentials rather than measurement error. The shift rates of in-plane mode under strain were calculated as $1.12 \mathrm{~cm}^{-1}$ per \% strain according to previous reports (Additional file 1: Fig. S6b) $[66,67]$. Therefore, the applied strain from surface fluctuations is about $0.17 \%$. Here the mini-strain is measured only at $120 \mathrm{~K}$. The peak shift of Raman spectra induced by mini-strain at $120 \mathrm{~K}$ is only $0.19 \mathrm{~cm}^{-1}$. In addition, the mini-strain arising from surface fluctuations has little effect on Raman spectra at room temperature. Therefore, it is challenging to map the temperature dependence of mini-strain. Considering the mini-strain is determined by the relative changes in spatial position, it can be speculated that the uniform temperature distribution has a much smaller influence on strain than the defects and impurities [68]. Consequently, we temporarily ignore the influence of temperature on mini-strain.

To assess the modulation of strain on exciton energy, strain engineered band structures of $\mathrm{WSe}_{2}$ monolayer were investigated by first-principle calculations. Band gaps with the highest valence bands (VB) and lowest conduction bands $(\mathrm{CB})$ and the trends of $\mathrm{K}-\mathrm{K}$ band gap of $\mathrm{WSe}_{2}$ monolayer under various biaxial strains, both compressive and tensile, are shown in Fig. $4 \mathrm{c}$ and d, respectively. The strong spin-orbit coupling (SOC) introduces spin splitting of hundreds $\mathrm{meV}$ in the $\mathrm{VB}$ and of a few to tens of $\mathrm{meV}$ in the $\mathrm{CB}$ at the $\mathrm{K}$ valley. The spin splitting results in an energy separation between bright excitons and dark excitons, and the dark excitons in $\mathrm{WSe}_{2}$ materials are predicted to be at lower energies than the bright ones. The biaxial strains have a much greater impact on the lowest CBs than the highest VBs. The direct band gap without strain is about $1.37 \mathrm{eV}$, which is generally underestimated for first-principle calculation. Under compression, the direct band gap of $\mathrm{K}-\mathrm{K}$ increases by about $100 \mathrm{meV}$ at $-1 \%$ strain, while the indirect band gap of $\mathrm{K}-\mathrm{Q}$ decreases. This results in a transition from direct to indirect band gap semiconductor. In contrast, under tensile the band gap decreases. The extracted $\mathrm{K}-\mathrm{K}$ bandgaps in Fig. 4d show that the band gap of $\mathrm{WSe}_{2}$ monolayer decreases/increases linearly with the slope of $-105.2 \pm 0.7 \mathrm{meV} / \%$ when tensile/compression is applied. In consequence, the modulation depth of disorder potentials arising from surface fluctuations is about $17.8 \mathrm{meV}$.

Considering phonon scattering and disorder potentials, the physical scenario of exciton nonequilibrium dynamics was established to explain the temperature-dependent exciton diffusion and PL spectra. As depicted in Fig. 4e, for the temperature higher than $280 \mathrm{~K}$, the scale of thermal fluctuation can be evaluated as $k_{\mathrm{B}} \mathrm{T}>26 \mathrm{meV}$, which is much larger than disorder potentials on the scale of 
$10 \mathrm{meV}$. Thus the phonon energy and density of states are large enough to facilitate excitons escape from disorder potentials. It means that phonons scattering dominates exciton diffusion rather than disorder potentials. Meanwhile, the phonons of high energy and density colliding with excitons can lead to exciton quenching effect and thus decreased PL efficiency. As the temperature is lowered, phonon energy and density of states decrease so that phonons effects are reduced. The disorder potentials dominate exciton dynamics and result in exciton localization, as shown in Fig. 4f. The localized exciton energy is determined by the modulation depth of disorder potential, thus the distribution of exciton energy has a bandwidth of $17 \mathrm{meV}$, which is proved by the exponential low-energy tail with a bandwidth of $14.8 \mathrm{meV}$ after deconvolution with RF (Fig. 3f).

It can be concluded that the competition between disorder-induced localization and phonons scattering creates a dilemma for extending exciton diffusion length. The optimized strategy is refrigerating the semiconductor to mitigate exciton-phonon scattering and suppressing the disorder to avoid exciton localization. The successful encapsulation with hBN can effectively suppress the disorder including local fluctuations of inherent material properties (such as chemical and structural composition, doping or strain) and external dielectric environment. Therefore, exciton diffusion experiments on $\mathrm{hBN}$ encapsulated 2D semiconductor were performed $[44,52,69,70]$, and the crucial role of disorder in exciton diffusion was verified. Naturally, we can expect a greater optimal diffusion coefficient in the hBN encapsulated 2D semiconductor at lower temperatures that exciton-phonon scattering is mitigated simultaneously.

\subsection{Exciton ultrafast dynamics dominated by phonon quenching and exciton localization}

The femtosecond pump-probe technique is a powerful tool to clarify the modulation of phonon quenching and exciton localization on the occupancy of exciton states within a femtosecond-picosecond timescale. In our ultrafast measurements, photon energy and energy density of pump pulse are $3.04 \mathrm{eV}$ and $10.0 \mu \mathrm{J} / \mathrm{cm}^{2}$, respectively. Considering the blue shift of exciton peak with decreasing temperature (Fig. 3c and d), photon energy and energy density of probe pulse were chosen as $1.65 \mathrm{eV}$ and $1.0 \mu \mathrm{J} / \mathrm{cm}^{2}$ for $220 \mathrm{~K}-310 \mathrm{~K}$, while chosen as $1.74 \mathrm{eV}$ and $0.2 \mu \mathrm{J} / \mathrm{cm}^{2}$ for $80 \mathrm{~K}-160 \mathrm{~K}$, respectively.

Figure 5 a depicts the differential reflection signals $\Delta R / R_{0}$ for different temperatures, which were normalized by globe maximum. The signals of $80 \mathrm{~K}-160 \mathrm{~K}$ were multiplied by 2 for clarity. Note that signals of $220 \mathrm{~K}-310 \mathrm{~K}$ reach their maximum at $260 \mathrm{~K}$, when the absorption peak approaches to probe photon energy of $1.74 \mathrm{eV}$. When temperature drops to the range of $80 \mathrm{~K}-160 \mathrm{~K}$, the $\Delta R / R_{0}$
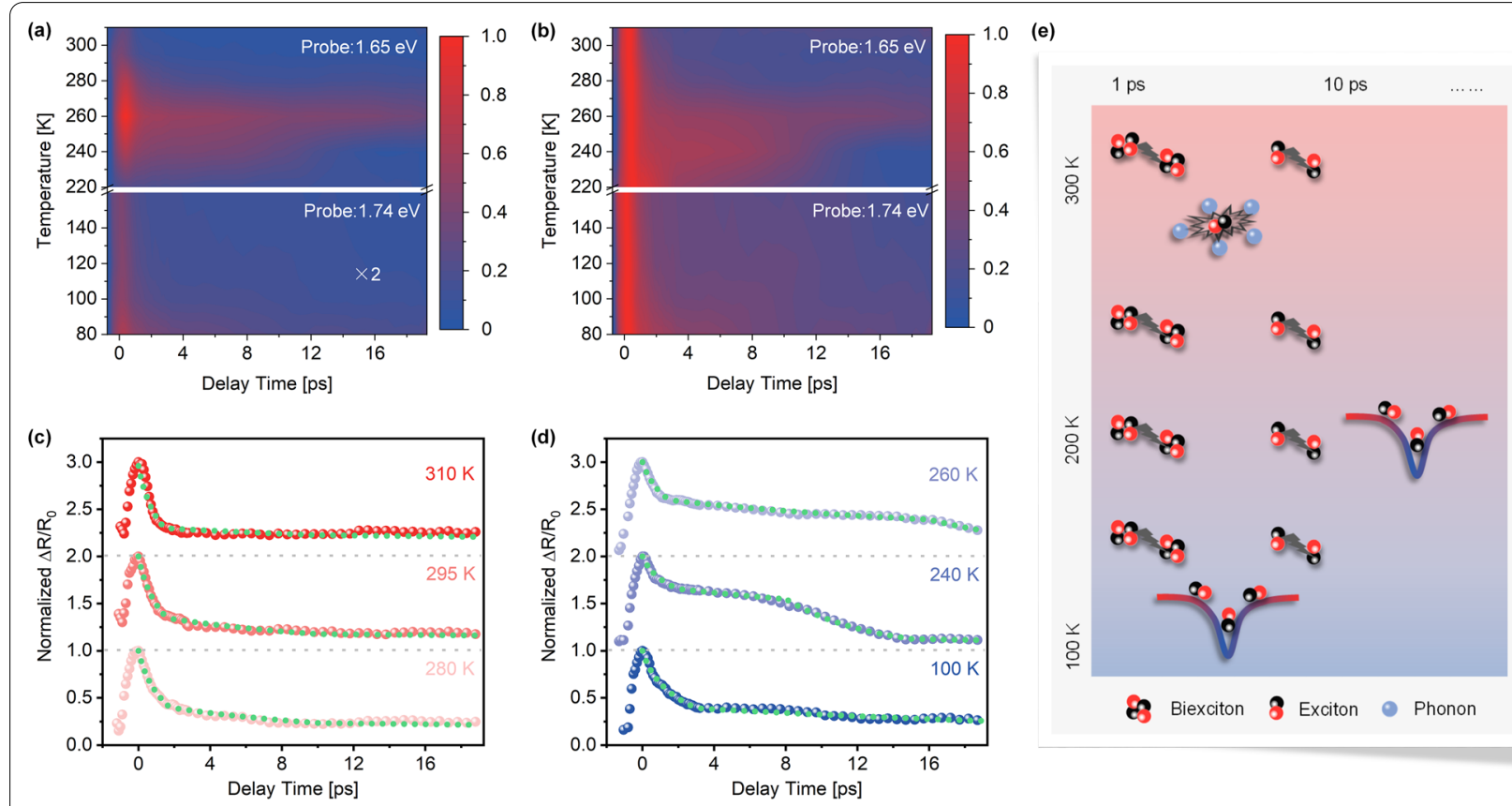

Fig. 5 Exciton relaxation dynamics dominated by phonon quenching and exciton localization. a Differential reflection signals $\Delta R / R_{0}$ for different temperatures, the peaks of $\Delta R / R_{0}$ were normalized by globe maximum and the signals of $80 \mathrm{~K}-160 \mathrm{~K}$ were multiplied by 2 for clarity. $\mathbf{b} U$ ltrafast signals in a normalized by the maximum of each temperature. $\mathbf{c}$, $\mathbf{d}$ Representative ultrafast signals extracted from $\mathbf{b}$. e Physical scenario of temperature dependent exciton relaxation dynamics. 
signals are much smaller than $220 \mathrm{~K}-310 \mathrm{~K}$ arising from two reasons: the relatively lower energy density of probe pulses for $80 \mathrm{~K}-160 \mathrm{~K}$ and the lowest dark state that can't couple with photons efficiently.

Focusing on the relaxation time of exciton, the ultrafast signals in Fig. 5a were normalized by the maximum of each temperature and presented in Fig. 5b. To quantitatively study exciton ultrafast dynamics modulated by phonon quenching and exciton localization, six representative ultrafast signals were extracted and sketched in Fig. 5c and d. In Fig. 5c, the ultrafast signals of $280 \mathrm{~K}$, $295 \mathrm{~K}$ and $310 \mathrm{~K}$ rapidly decay to $\sim 20 \%$, which is consistent with previous measurements at room temperature [23]. However, the ultrafast signals of $260 \mathrm{~K}$ in Fig. $5 \mathrm{~d}$ just drop down to $\sim 50 \%$ in the first 2 ps, which means there is a relaxation channel weaken or even disappear, considering the strength of $\Delta R / R_{0}$ is proportional to the pumped exciton density [71]. Moreover, the ultrafast signal of $240 \mathrm{~K}$ continues to decline to $\sim 10 \%$ after the first 8 ps, as shown in the middle of Fig. $5 \mathrm{~d}$. It implies that a new exciton relaxation channel appears at $8 \mathrm{ps}$. When temperature drops to $100 \mathrm{~K}$, ultrafast signal (bottom of Fig. 5d) rapidly decays to $\sim 20 \%$ again, similar to Fig. $5 \mathrm{c}$.

Consistent with diffusion dynamics and PL spectra, phonon quenching and exciton localization can provide a reasonable interpretation for temperature-dependent relaxation dynamics of excitons. Generally, the high density excitons pumped by femtosecond pulses (the rising edge of $\Delta R / R_{0}$ ) undergo two decay processes before 10 ps: biexciton-biexciton annihilation $\left(\sim N^{4}\right)$, excitonexciton annihilation $\left(\sim N^{2}\right)$ [72]. Integrating phonon quenching and exciton localization into the common rate equation, the modified rate equation that describes the ultrafast interaction dynamics among excitons, phonons and localized excitons can be written as following:

$$
\frac{\partial N}{\partial t}=-\alpha N^{4}-\beta N^{2}-\sigma_{p}(T) N-\sigma_{d}\left(T, t>t_{s}\right) N
$$

where $N$ is exciton density, $\alpha$ and $\beta$ are biexciton-biexciton annihilation rate and exciton-exciton annihilation rate, $\sigma_{p}(T)$ is exciton decay rate of multi-phonons quenching, and $\sigma_{d}\left(T, t>t_{s}\right)$ is transition rate from free exciton to localized exciton owing to disorder potentials, which is related to temperature and delay time, and $t_{s}$ refers to the starting time of exciton localization. It should be noted that diffusion and radiative recombination of exciton can be safely disregarded on this time scale $[21,23,26,73]$. Seemingly, the third and fourth terms describing the multi-phonons quenching and exciton localization cannot be distinguished. However, the time dominating exciton decay for phonon quenching and exciton localization is temperature dependent, so they were separated into temperature dependent two terms in Eq. (2). The differential Eq. (2) was numerically solved using the Runge-Kutta method, and the ultrafast signals at different temperatures were faithfully reproduced by the modified rate equation, as depicted by green dotted curves in Fig. $5 \mathrm{c}$ and d. The fitted parameters were summarized in Additional file 1: Table S1.

The physical scenario of temperature-dependent exciton relaxation dynamics was schematically summarized in Fig. 5e. For the temperatures of $280 \mathrm{~K}, 295 \mathrm{~K}$ and $310 \mathrm{~K}$, the exciton relaxation is dominated by biexciton-biexciton annihilation, exciton-exciton annihilation and phonon quenching. The phonon quenching substantially weakens at $260 \mathrm{~K}$, while exciton localization appears prominently at $8 \mathrm{ps}$ for $240 \mathrm{~K}$, which is about $16 \mathrm{ps}$ for $260 \mathrm{~K}$. Therefore, it can be expected that the onset of exciton localization brings forward as decreasing temperature until keeping up with the multi-excitons annihilation and eventually results in the rapid decay of ultrafast signal during $0-4 \mathrm{ps}$ at $100 \mathrm{~K}$.

\section{Conclusions}

In conclusion, the diffusion and relaxation of $2 \mathrm{D}$ excitons modulated by phonon scattering and disorder potentials were investigated with micro-PL imaging, PL spectra and femtosecond pump probe techniques. As increasing temperature, the exciton diffusion length and PL intensity increase initially and then decrease and reach maximum at $280 \mathrm{~K}$, where the effective diffusion coefficient was enhanced by $>200 \%$, and the DOA of PL spectra decreases rapidly from $25 \%$ to nearly $0 \%$ with an exponential trend. These phenomena were explained by a selfconsistent physical scenario of exciton nonequilibrium dynamics modulated by phonon scattering and disorder potentials. Moreover, the ultrafast relaxation of excitons can be effectively modulated by the phonon quenching and exciton localization with temperature variation. It indicates that disorder potentials are crucial to exciton dynamics at cryogenic temperature. This work can deepen our understanding of 2D exciton nonequilibrium dynamics including spatial diffusion and temporal relaxation dominated by phonon scattering and exciton localization, and provides us an important implication for long-range exciton diffusion and thermal management, thereby paving the way to functional excitonic devices. Additionally, the investigation of $2 \mathrm{D}$ exciton localization in disorder potentials is of great significance for manipulating excitons and realizing promising phenomena such as exciton valley hall effect $[74,75]$. Anderson localization and exciton condensation. 


\section{Methods}

\subsection{Sample preparations}

The two-dimensional crystal was mechanically exfoliated from the bulk $\mathrm{WSe}_{2}$ by the commercial scotch tape (USI Adhesive Plastic Film), and then the thinner $\mathrm{WSe}_{2}$ was exfoliated and transferred by the transparent polydimethylsiloxane (PDMS) film. The $\mathrm{WSe}_{2}$ monolayer can be determined by the optical microscope. Raman spectrum and AFM characterization can also be carried out to confirm the thickness of the sample flake. The PDMS with the attached identified flake was pressed against the target $\mathrm{Si} / \mathrm{SiO}_{2}$ substrate, and then peeled off very slowly to transfer the $\mathrm{WSe}_{2}$ monolayer to the substrate.

\subsection{Confocal Raman and PL spectrum measurements}

Raman and PL Mappings were performed by a confocal micro-Raman spectrometer (Horiba XploRA PLUS), employing an excitation laser with a wavelength of $532 \mathrm{~nm}$. The Raman spectra were detected with a $100 \times$ objective lens (Olympus MPlan N, NA=0.9) and $1800 \mathrm{~mm}^{-1}$ grating for the high spatial and spectral resolution $\left(\sim 1.8 \mathrm{~cm}^{-1}\right)$. While for the PL spectrum, $600 \mathrm{~mm}^{-1}$ grating was used for high intensity. The step size of the PL and Raman image was set as $0.6 \mu \mathrm{m} \times 0.6 \mu \mathrm{m}$.

\subsection{Micro PL imaging}

The schematic of micro PL imaging apparatus was presented in Additional file 1: Fig. S7. The femtosecond pulses $(817 \mathrm{~nm}, 73 \mathrm{fs}, 80 \mathrm{MHz}$ ) emitted from modelocked oscillator (Tsunami 3941C-25XP) were focused into a photonic crystal fiber (Newport SCG-800) to generate the super-continuum white light. The laser pulses were selected by $600 \pm 10 \mathrm{~nm}$ bandpass filter (Thorlabs FB600-10) and then focused by an infinity-corrected long work distance micro-objective (Mitutoyo, $100 \times$ ) to excite the $\mathrm{WSe}_{2}$ monolayer. Then a $650 \mathrm{~nm}$ long-pass edge filter (Thorlabs FEL0650) was used to block excitation light, the filtered light was imaged by an EMCCD camera (Andor Ixon 888) with the micro objective and matched widefield tube lens (Thorlabs TTL200-A), and the spatial resolution is $0.6 \mu \mathrm{m}$. For spectral measurements, the filtered light was coupled to a spectrometer (Acton SP2500, spectral resolution is $0.05 \mathrm{~nm}$, phonon energy resolution is about $0.1 \mathrm{meV}$ for $750 \mathrm{~nm}$ ) equipped with a liquid nitrogen cooled CCD. The homemade apparatus is convenient for switching between spectral measurement and spatial imaging by the removable right-angle silver mirror in cage cube.

\subsection{Ultrafast measurements}

Ultrafast pump probe measurements in reflection configuration were carried out. The femtosecond pulses
(817 nm, $73 \mathrm{fs}, 80 \mathrm{MHz}$ ) were split into two parts. One of them passed through a BBO crystal to produce the $408 \mathrm{~nm}$ pump pulses, while the other one was focused into a photonic crystal fiber (Newport SCG-800) to generate the super-continuum white light. The probe was then selected with a $710 \pm 10 \mathrm{~nm}$ (Thorlabs FB71010) and $750 \pm 10 \mathrm{~nm}$ (Thorlabs FB750-10) bandpass filters for $80 \mathrm{~K}-160 \mathrm{~K}$ and $220 \mathrm{~K}-310 \mathrm{~K}$, respectively. The spot size of the focused probe and pump laser was $<1 \mu \mathrm{m}$ (See Additional file 1: S9 for more details). The delay time between pump and probe pulses was controlled by a steeper linear stage (Newport M-ILS150PP). To improve the signal-to-noise ratio, the reflected probe pulses passed through a $450 \mathrm{~nm}$ longpass edge filter (Thorlabs FEL0450) and then were detected by a high-sensitivity photomultiplier (Thorlabs PMM02) connected with the phase lock-in amplifier (Stanford SR830).

\subsection{Numerical simulations}

The first-principles calculations were performed using the Vienna Ab initio Simulation Package based on plane waves and the projector augmented wave method. The Perdew-Burke-Ernzerhof exchange-correlation functional was used for all calculations, and the vdW interactions were considered in the DFT-D2 method. Keeping the in-plane positions fixed, the outof-plane positions were relaxed for all atoms until the energy difference of successive atom configurations was less than $10^{-6} \mathrm{eV}$. The out-of-plane force on each atom in the relaxed structure was less than $0.001 \mathrm{eV} / \AA$. The cutoff energy of the plane-wave basis was set to $500 \mathrm{eV}$, and the convergence criterion for total energy was $10^{-8} \mathrm{eV}$. A $\Gamma$-centered $\mathrm{k}$ mesh of $15 \times 15 \times 1$ was used for both the relaxation and normal calculations. The thickness of the vacuum layer was greater than $20 \AA$ to avoid impacts from neighboring periodic images. SOC was taken into account for all calculations, except in structure relaxation.

\section{Supplementary Information}

The online version contains supplementary material available at https://doi. org/10.1186/s43593-021-00006-8.

Additional file 1: Figure S1. PL images of WSe ${ }_{2}$ monolayer for different temperatures. Figure S2. Extracting effective diffusion coefficients. Figure S3. Temperature-dependent photoluminescence model. Figure S4. Temperature-dependent photoluminescence spectra. Figure S5. Excitation power-dependent photoluminescence spectra at $80 \mathrm{~K}$ and $320 \mathrm{~K}$. Figure S6. Calculation of strain from Raman mappings at $120 \mathrm{~K}$. Figure S7. Fitting of exciton ultrafast relaxation. Figure S8. Schematic of Micro PL imaging apparatus. Figure $\mathbf{5 9}$. Spot Size of focused probe and pump laser. 


\section{Acknowledgements}

The first-principles calculations is supported by High-performance Computing Platform of Peking University.

\section{Authors' contributions}

ZF supervised the project. PQ and YL performed all experiments. BBS completed the first-principles calculations. YL, WL, and PQ carried out photoluminescence and Raman mappings. PQ established the theoretical model and wrote the paper. All authors contribute to the scientific discussions and paper revisions. All authors read and approved the final manuscript.

\section{Funding}

National Key Research and Development Program of China (Grant No. 2017YFA0206000), Beijing Natural Science Foundation (Grant No. Z180011), National Science Foundation of China (Grant Nos. 12027807, 12104241 and 61521004), Project funded by China Postdoctoral Science Foundation (Grant No. 2019M660283).

\section{Availability of data and materials}

All data generated or analysed during this study are included in this published article (and its additional files). Additional data related to this paper may be requested from the authors.

\section{Declarations}

\section{Competing interests}

The authors declare that they have no competing interests.

\section{Author details}

${ }^{1}$ School of Physics, State Key Laboratory for Mesoscopic Physics, Academy for Advanced Interdisciplinary Studies, Collaborative Innovation Center of Quantum Matter, Nano-Optoelectronics Frontier Center of Ministry of Education, Peking University, Beijing 100871, China. ${ }^{2}$ Institute of Modern Optics, Tianjin Key Laboratory of Micro-Scale Optical Information Science and Technology, Nankai University, Tianjin 300350, China. ${ }^{3}$ Beijing Key Laboratory for Magnetoelectric Materials and Devices, Beijing Innovation Centre for Engineering Science and Advanced Technology, Department of Materials Science and Engineering, College of Engineering, Peking University, Beijing 100871, China.

Received: 13 April 2021 Revised: 4 October 2021 Accepted: 5 October 2021

Published online: 08 November 2021

\section{References}

1. T. Kato, T. Kaneko, Transport Dynamics of Neutral Excitons and Trions in Monolayer WS 2 . ACS Nano 10, 9687-9694 (2016). https://doi.org/10. 1021/acsnano.6b05580

2. A.G. Milnes, Semiconductor Devices and Integrated Electronics (Springer, New York, 2012), pp. 505-507

3. Q. Xu, B. Schmidt, S. Pradhan, M. Lipson, Micrometre-scale silicon electrooptic modulator. Nature 435, 325-327 (2005)

4. G. Moody, J. Schaibley, X. Xu, Exciton dynamics in monolayer transition metal dichalcogenides. J. Opt. Soc. Am. B: Opt. Phys. 33, C39-C49 (2016)

5. A. Chernikov et al., Exciton binding energy and nonhydrogenic rydberg series in monolayer WS2. Phys. Rev. Lett. 113, 076802 (2014)

6. M.M. Ugeda et al., Giant bandgap renormalization and excitonic effects in a monolayer transition metal dichalcogenide semiconductor. Nat. Mater. 13, 1091-1095 (2014)

7. K. He et al., Tightly bound excitons in monolayer WSe 2 . Phys. Rev. Lett. 113, 026803 (2014). https://doi.org/10.1103/PhysRevLett.113.026803

8. T. Lundstrom, W. Schoenfeld, H. Lee, P. Petroff, Exciton storage in semiconductor self-assembled quantum dots. Science 286, 2312-2314 (1999)

9. A. Gärtner, A. Holleitner, J. Kotthaus, D. Schuh, Drift mobility of long-living excitons in coupled GaAs quantum wells. Appl. Phys. Lett. 89, 052108 (2006)
10. A.G. Winbow, A.T. Hammack, L.V. Butov, A.C. Gossard, Photon storage with nanosecond switching in coupled quantum well nanostructures. Nano Lett. 7, 1349-1351 (2007)

11. A. High, A. Hammack, L. Butov, M. Hanson, A. Gossard, Exciton optoelectronic transistor. Opt. Lett. 32, 2466-2468 (2007)

12. A.A. High, E.E. Novitskaya, L.V. Butov, M. Hanson, A.C. Gossard, Control of exciton fluxes in an excitonic integrated circuit. Science 321, 229-231 (2008)

13. G. Grosso et al., excitonic switches operating at around $100 \mathrm{~K}$. Nat. Photonics 3, 577-580 (2009)

14. M. Seitz et al., Exciton diffusion in two-dimensional metal-halide perovskites. Nat. Commun. 11, 2035 (2020). https://doi.org/10.1038/ s41467-020-15882-w

15. Y. Firdaus et al., Long-range exciton diffusion in molecular non-fullerene acceptors. Nat. Commun. 11, 5220 (2020). https://doi.org/10.1038/ s41467-020-19029-9

16. S. Deng et al., Long-range exciton transport and slow annihilation in twodimensional hybrid perovskites. Nat. Commun. 11, 664 (2020). https:// doi.org/10.1038/s41467-020-14403-z

17. J. Feng, X. Qian, C.-W. Huang, J. Li, Strain-engineered artificial atom as a broad-spectrum solar energy funnel. Nat. Photonics 6, 866-872 (2012). https://doi.org/10.1038/nphoton.2012.285

18. H. Li et al., Optoelectronic crystal of artificial atoms in strain-textured molybdenum disulphide. Nat. Commun. 6, 7381 (2015)

19. D. Unuchek et al., Room-temperature electrical control of exciton flux in a van der waals heterostructure. Nature 560, 340-344 (2018)

20. D. Unuchek et al., Valley-Polarized Exciton Currents in a Van Der Waals Heterostructure. Nat. Nanotechnol. 1, 1104-1109 (2019)

21. C. Jin et al., Imaging of pure spin-valley diffusion current in $\mathrm{WS}_{2}-\mathrm{WSe}_{2}$ heterostructures. Science 360, 893-896 (2018)

22. S. Hao, M.Z. Bellus, D. He, Y. Wang, H. Zhao, Controlling exciton transport in monolayer MoSe $_{2}$ by dielectric screening. Nanoscale Horiz. 5, 139-143 (2019)

23. P. Qi et al., Remote lightening and ultrafast transition: intrinsic modulation of exciton spatiotemporal dynamics in monolayer MoS2. ACS Nano 14, 6897-6905 (2020). https://doi.org/10.1021/acsnano.0c01165

24. L. Yuan et al., Twist-angle-dependent interlayer exciton diffusion in WS2WSe2 heterobilayers. Nat. Mater. 19, 617-623 (2020). https://doi.org/10. 1038/s41563-020-0670-3

25. J. Choi et al., Moiré potential impedes interlayer exciton diffusion in van der Waals heterostructures. Sci. Adv. 6, eaba8866 (2020). https://doi.org/ 10.1126/sciadv.aba8866

26. Kulig, M. et al. Exciton Diffusion and Halo Effects in Monolayer Semiconductors. Phys. Rev. Lett. 120, 207401 (2018).

27. E.V. Castro et al., Limits on charge carrier mobility in suspended graphene due to flexural phonons. Phys. Rev. Lett. 105, 266601 (2010). https://doi. org/10.1103/PhysRevLett.105.266601

28. J. Li et al., Phonon-limited carrier mobility and resistivity from carbon nanotubes to graphene. Phys. Rev. B 92, 075414 (2015). https://doi.org/ 10.1103/PhysRevB.92.075414

29. J.-J. Zhou, M. Bernardi, Ab initio electron mobility and polar phonon scattering in GaAs. Phys. Rev. B 94, 201201 (2016). https://doi.org/10.1103/ PhysRevB.94.201201

30. T. Fang, A. Konar, H. Xing, D. Jena, Mobility in semiconducting graphene nanoribbons: Phonon, impurity, and edge roughness scattering. Phys. Rev. B 78, 205403 (2008). https://doi.org/10.1103/PhysRevB.78.205403

31. T. Deng et al., EPIC STAR: a reliable and efficient approach for phononand impurity-limited charge transport calculations. NPJ Comput. Mater. 6 46 (2020). https://doi.org/10.1038/s41524-020-0316-7

32. Y. Huang et al., Reliable exfoliation of large-area high-quality flakes of graphene and other two-dimensional materials. ACS Nano 9, 10612-10620 (2015). https://doi.org/10.1021/acsnano.5b04258

33. W. Bao et al., Visualizing nanoscale excitonic relaxation properties of disordered edges and grain boundaries in monolayer molybdenum disulfide. Nat. Commun. 6, 7993 (2015)

34. M.S. Kim et al., Biexciton emission from edges and grain boundaries of triangular WS 2 monolayers. ACS Nano 10, 2399-2405 (2016)

35. G.G. Naumis, S. Barraza-Lopez, M. Oliva-Leyva, H. Terrones, Electronic and optical properties of strained graphene and other strained 2D materials: a 
review. Rep. Prog. Phys. 80, 096501 (2017). https://doi.org/10.1088/13616633/aa74ef

36. G.J. Verbiest, C. Stampfer, S.E. Huber, M. Andersen, K. Reuter, Interplay between nanometer-scale strain variations and externally applied strain in graphene. Phys. Rev. B 93, 195438 (2016). https://doi.org/10.1103/ PhysRevB.93.195438

37. R. Maiti et al., Strain-engineered high-responsivity MoTe2 photodetector for silicon photonic integrated circuits. Nat. Photonics 14, 578-584 (2020). https://doi.org/10.1038/s41566-020-0647-4

38. M.G. Harats, J.N. Kirchhof, M. Qiao, K. Greben, K.I. Bolotin, Dynamics and efficient conversion of excitons to trions in non-uniformly strained monolayer WS2. Nat. Photonics 14, 324-329 (2020). https://doi.org/10.1038/ s41566-019-0581-5

39. T.P. Darlington et al., Imaging strain-localized excitons in nanoscale bubbles of monolayer WSe2 at room temperature. Nat. Nanotechnol. 15, 854-860 (2020). https://doi.org/10.1038/s41565-020-0730-5

40. X. Zhang et al., Phonon and Raman scattering of two-dimensional transition metal dichalcogenides from monolayer, multilayer to bulk material. Chem Soc Rev 44, 2757-2785 (2015). https://doi.org/10.1039/c4cs0 0282b

41. B.R. Carvalho, M.A. Pimenta, Resonance Raman spectroscopy in semiconducting transition-metal dichalcogenides: basic properties and perspectives. 2D Materials (2020). https://doi.org/10.1088/2053-1583/ab98ef

42. F. Cadiz et al., Exciton diffusion in WSe2 monolayers embedded in a van der Waals heterostructure. Appl. Phys. Lett. 112, 152106 (2018). https:// doi.org/10.1063/1.5026478

43. R. Perea-Causín et al., Exciton propagation and halo formation in twodimensional materials. Nano Lett. 19, 7317-7323 (2019). https://doi.org/ 10.1021/acs.nanolett.9b02948

44. J. Zipfel et al., Exciton diffusion in monolayer semiconductors with suppressed disorder. Phys. Rev. B 101, 115430 (2020). https://doi.org/10. 1103/PhysRevB.101.115430

45. D. Sun et al., Observation of rapid exciton-exciton annihilation in monolayer molybdenum disulfide. Nano Lett. 14, 5625-5629 (2014). https:// doi.org/10.1021/nl5021975

46. N. Kumar et al., Exciton-exciton annihilation in MoSe2 monolayers. Phys. Rev. B 89, 125427 (2014). https://doi.org/10.1 103/PhysRevB.89.125427

47. S. Mouri et al., Nonlinear photoluminescence in atomically thin layeredWSe2arising from diffusion-assisted exciton-exciton annihilation. Phys. Rev. B 90, 155449 (2014). https://doi.org/10.1103/PhysRevB.90.155449

48. T. Xia, D.J. Hagan, M. Sheik-Bahae, E.W. Van Stryland, Eclipsing Z-scan measurement of $\lambda / 104$ wave-front distortion. Opt. Lett. 19, 317-319 (1994). https://doi.org/10.1364/OL.19.000317

49. P. Qi et al., Optical nonlinearities of alcoholic liquids under high-repetition-rate femtosecond lasers by single beam time-resolved eclipsed Z-scan. Opt. Laser Technol. 109, 643-647 (2019). https://doi.org/10.1016/j. optlastec.2018.08.054

50. X.-X. Zhang, Y. You, S.Y.F. Zhao, T.F. Heinz, Experimental Evidence for Dark Excitons in Monolayer WSe 2 . Phys. Rev. Lett. 115, 257403 (2015). https:// doi.org/10.1103/PhysRevLett.115.257403

51. M.M. Glazov, Quantum interference effect on exciton transport in monolayer semiconductors. Phys. Rev. Lett. 124, 166802 (2020). https://doi. org/10.1103/PhysRevLett.124.166802

52. A. Raja et al., Dielectric disorder in two-dimensional materials. Nat. Nanotechnol. 14, 832-837 (2019). https://doi.org/10.1038/s41565-019-0520-0

53. M.M. Glazov, Phonon wind and drag of excitons in monolayer semiconductors. Phys. Rev. B 100, 045426 (2019). https://doi.org/10.1103/PhysR evB.100.045426

54. Y.P. Varshni, Temperature dependence of the energy gap in semiconductors. Physica 34, 149-154 (1967). https://doi.org/10.1016/0031-8914(67) 90062-6
55. D. Christiansen et al., Phonon sidebands in monolayer transition metal dichalcogenides. Phys. Rev. Lett. 119, 187402 (2017). https://doi.org/10. 1103/PhysRevLett.119.187402

56. A. Raja et al., Enhancement of exciton-phonon scattering from monolayer to bilayer WS2. Nano Lett. 18,6135-6143 (2018). https://doi.org/10.1021/ acs.nanolett.8b01793

57. S. Helmrich et al., Exciton-phonon coupling in mono- and bilayer MoTe 2 . 2D Mater. 5, 045007 (2018). https://doi.org/10.1088/2053-1583/aacfb7

58. H. Li, X. Zhu, Z.K. Tang, X.H. Zhang, Low-temperature photoluminescence emission of monolayer MoS2 on diverse substrates grown by CVD. J. Lumin. 199, 210-215 (2018). https://doi.org/10.1016/j.jlumin.2018.03.052

59. S. Rudin, T.L. Reinecke, B. Segall, Temperature-dependent exciton linewidths in semiconductors. Phys. Rev. B 42, 11218-11231 (1990). https://doi.org/10.1103/physrevb.42.11218

60. M. Selig et al., Excitonic linewidth and coherence lifetime in monolayer transition metal dichalcogenides. Nat. Commun. 7, 13279 (2016). https:// doi.org/10.1038/ncomms13279

61. S. Brem et al., Phonon-assisted photoluminescence from indirect excitons in monolayers of transition-metal dichalcogenides. Nano Lett 20, 2849-2856 (2020). https://doi.org/10.1021/acs.nanolett.0c00633

62. Jansson, P. A. Deconvolution of images and spectra. (Courier Corporation, 2014).

63. M. Jones et al., Analysis of photoluminescence from solubilized singlewalled carbon nanotubes. Phys. Rev. B (2005). https://doi.org/10.1103/ PhysRevB.71.115426

64. S. Repp, S. Weber, E. Erdem, Defect evolution of nonstoichiometric ZnO quantum dots. J. Phys. Chem. C 120, 25124-25130 (2016). https://doi. org/10.1021/acs.jpcc.6b09108

65. H.J. Conley et al., Bandgap engineering of strained monolayer and bilayer MoS2. Nano Lett. 13, 3626 (2013)

66. B. Liu et al., Strain-engineered van der waals interfaces of mixed-dimensional heterostructure arrays. ACS Nano 13, 9057-9066 (2019). https:// doi.org/10.1021/acsnano.9b03239

67. D. Lloyd et al., Band gap engineering with ultralarge biaxial strains in suspended monolayer MoS2. Nano Lett 16, 5836-5841 (2016). https:// doi.org/10.1021/acs.nanolett.6b02615

68. K. Shibata, J. Yan, Y. Hazama, S. Chen, H. Akiyama, Exciton localization and enhancement of the exciton-LO phonon interaction in a $\mathrm{Cs} \mathrm{PbBr} 3$ single crystal. J. Phys. Chem. C 124, 18257-18263 (2020). https://doi.org/10. 1021/acs.jpcc.0c06254

69. J.D. Ziegler et al., Fast and anomalous exciton diffusion in two-dimensional hybrid perovskites. Nano Lett. 20, 6674-6681 (2020). https://doi. org/10.1021/acs.nanolett.0c02472

70. Z. Li et al., Interlayer exciton transport in MoSe2/WSe2 heterostructures. ACS Nano (2021). https://doi.org/10.1021/acsnano.0c08981

71. H. Shan et al., Direct observation of ultrafast plasmonic hot electron transfer in the strong coupling regime. Light. 8, 9 (2019)

72. H. Shan et al., Electron transfer and cascade relaxation dynamics of graphene quantum dots/MoS2 monolayer mixed-dimensional van der Waals heterostructures. Mater. Today 24, 10-16 (2019). https://doi.org/10. 1016/j.mattod.2019.01.015

73. C. Jin et al., On Optical dipole moment and radiative recombination lifetime of excitons in WSe2. Adv. Funct. Mater. 27, 1601741 (2017). https:// doi.org/10.1002/adfm.201601741

74. Z. Huang et al., Robust room temperature valley Hall effect of interlayer excitons. Nano Lett. 20, 1345-1351 (2019)

75. Y. Liu et al., Electrically controllable router of interlayer excitons. Sci. Adv. 6, eaba1830 (2020) 